\title{
Desynchronization Waves in Power Grids Described by a Kuramoto-like Model
}

\author{
Xuewei Zhang, Markus Zahn \\ Department of Electrical Engineering and Computer Science, Massachusetts Institute of \\ Technology, Cambridge, Massachusetts, USA
}

\begin{abstract}
A network of coupled Kuramoto-like oscillators is studied to model the dynamics in modern power systems. While previous works focus on the criteria of synchronization, we in this paper study the development of desynchronization, which may lead to power outrage and system breakdown in real power systems. Instead of numerically simulating detailed component-level models, we use a simplified network-reduced model which captures essential features of a real system, including spread of desynchronization waves under disturbances. Some statistical results about the relation between the maximum disturbance and coupling strength are also found.
\end{abstract}

Keywords: Power system stability, Complex networks, Kuramoto-like model, Desynchronization

\section{Introduction}

Recently, the problems of synchronization and transient stability in power grids have been analyzed using Kuramoto-like models [1-3]. In these works, the original Kuramoto oscillator, a paradigm in modern complex networks research $[4,5]$, has been modified to describe the dynamics in simplified power systems. Essentially, the stable operation of power grids, like the synchronization of coupled Kuramoto oscillators, is to maintain a phase-locked state of the whole system [6]. In addition to control at the power generation end, which is currently used to achieve synchronization, the development of smart grid $[7,8]$ extends the control to the consumer side to cope with the more distributed supply in future. Ultimately, numerical simulations of detailed componentlevel models are necessary for each specific case. However, simplified modeling the power grids with interconnected nonlinear oscillators may help to obtain some general insights on the network level.

The organization of this paper is as follows. In Section 2, the derivation of the Kuramoto-like oscillator networks as a power grid model is outlined following Ref. $[1,2]$. We also examine the existence of synchronized state in two simplest cases with two or three nodes. In Section 3, we consider a ring of identical dynamical units with varying coupling strength. The master stability analysis [9] is applied to estimate the onset of desynchronization, which will then spread to the whole system like a wave as indicated by numerical results. Some comments and conclusions are drawn in Section 4.

\section{The Model}

Consider a network of $N$ synchronous generators (production) and motors (load). The state of element $j$ is described by its phase $\theta_{j}=\Omega t+\tilde{\theta}_{j}$ and phase ty $\dot{\theta}_{j}=\Omega+\dot{\tilde{\theta}}_{j}$, where $\Omega$ is the grid frequency. Note that the elements here may refer to either a real generator/motor or an 
equivalent representation of a sub-grid. The latter is called network reduction, the first step toward understanding dynamical processes on complex networks.

The generated power $P_{s r c, j}$ of element $j$ must balance the power transmitted to the grid $P_{\text {trans, } j}$ plus that for local acceleration $P_{a c c, j}=(I / 2) d \dot{\theta}_{j}^{2} / d t$ and dissipation $P_{d i s s, j}=K \dot{\theta}_{j}{ }^{2}$, where $K$, the friction coefficient, and $I$, the moment of inertia, are assumed to be uniform across the network in this paper.

Assuming that $\theta_{j} \ll \Omega$, to the first order approximation, one has $P_{a c c, j} \approx I \Omega \ddot{\tilde{\theta}}_{j}$ and $P_{\text {diss }, j} \approx K\left(\Omega^{2}+2 \dot{\tilde{\theta}}_{j} \Omega\right)$. If the capacity (maximum transmission power) of the line connecting two elements $j$ and $i$ is $P_{\max , j i}\left(=P_{\max , i j}\right)$, the power flow from $j$ to $i$ is then $P_{\max , j i} \sin \left(\theta_{j}-\theta_{i}\right)$, or equivalently, $P_{\max , j i} \sin \left(\tilde{\theta}_{j}-\tilde{\theta}_{i}\right)$. When $j$ and $i$ are not connected, we simply set $P_{\max , j i}=0$.

The equation of motion is thus:

$$
\ddot{\widetilde{\theta}}_{j}=P_{j}-\alpha \dot{\tilde{\theta}}_{j}-\sum_{i} k_{j i} \sin \left(\tilde{\theta}_{j}-\tilde{\theta}_{i}\right)
$$

where $P_{j}=\left(P_{s r c, j}-K \Omega^{2}\right) /(I \Omega)$ represents the generation capability of element $j, \alpha=2 K / I$ is an effective damping coefficient and $k_{j i}=P_{\max , j i} /(I \Omega)$ encodes both coupling strength and connectivity. When the acceleration term, i.e. left side of Eq. (1), is negligible, the system is reduced to the Kuramoto model.

In synchronized state, all $N$ elements work at the grid frequency and the steady-state solution of Eq. (1) is a set of constant phases $\left\{\hat{\theta}_{j}\right\}_{j=1}^{N}$. A necessary condition for this is $\sum_{j} P_{j}=0$, that is, total generated power balances load.

When $N=2$, and set $P_{1}=-P_{2}=P$, $\hat{\theta}_{2}=0, k_{12}=k_{21}=k$, then there is a globally stable fixed point at $\hat{\theta}_{1}=\arcsin (P / k)$ for $P<k$. Otherwise, the phase difference is shown to converge to a limit cycle under weak damping (small $\alpha$ ) [1].

When $N=3$ under a chain configuration, $k_{12}=k_{21}=k, k_{32}=k_{23}=b k$, $k_{13}=k_{31}=0, P_{2}=P, P_{1}=-c P$, $P_{3}=(c-1) P$, where $c$ and $b$ are parameters. A fixed point of the system is: $\hat{\theta}_{2}=0, \hat{\theta}_{1}=\arcsin (-c P / k), \hat{\theta}_{3}=$ $\arcsin ((c-1) P / b k)$ for certain range of the parameters. In Fig. 1, we show the region of synchronization in the parameter space. Note that $P<k$ is not required for the existence of fixed points.

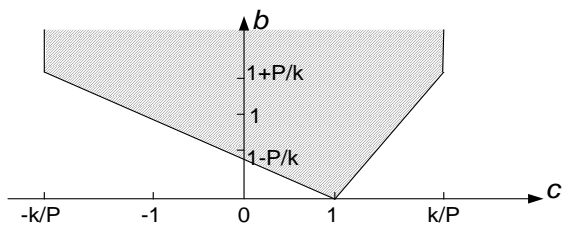

(a). $P / k<1$

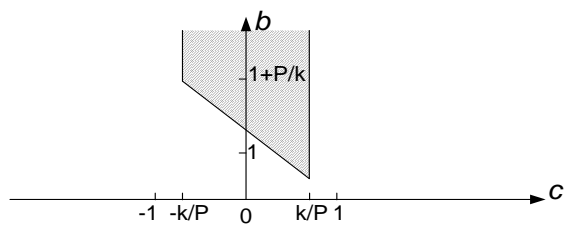

(b). $P / k>1$

Fig. 1: The synchronization region in 3-node chain with (a) $P / k<1$ and (b) $P / k>1$.

\section{Desynchronization Waves}

The main conclusions of previous works state that when the coupling strength is high enough, a self-organized synchronization will be approached in the system. However, an unanswered question is: starting from a synchronized state, how does the desynchronization develop? Intuitively, if there is a disturbance at a node, it will deviate from the steady state and "drag" its neighbors away from their original states, which will affect more adjacent nodes in turn. Under certain condi- 
tions, this cascade can spread over the whole network without significant decay.

Quantitatively, perform the small signal perturbation of Eq. (1):

$$
\ddot{\delta}_{j}=-\alpha \dot{\delta}_{j}-\sum_{i} K_{j i} \cos \left(\hat{\theta}_{j}-\hat{\theta}_{i}\right) \delta_{i}
$$

where $\delta_{j}$ is the small deviation from the steady state $\hat{\theta}_{j}, K_{j i}=-k_{j i}$ when $i \neq j$, and $K_{j j}=\sum_{i \neq j} k_{j i}$.

Now consider a special case: let $K_{j i} \cos \left(\hat{\theta}_{j}-\hat{\theta}_{i}\right)=k G_{j i}$, where constant $k$ is the global strength of coupling and the matrix $G$ is a symmetric Laplacian, i.e. $G_{j i}=G_{i j}$ and $\sum_{i} G_{j i}=0$. Further, the $N$ elements are arranged in a ring configuration with nearest-neighbor couplings. The form of the connectivity matrix $G$ is:

$$
G=\left[\begin{array}{ccccccc}
a_{1} & b_{1} & 0 & 0 & \cdots & 0 & b_{N} \\
b_{1} & a_{2} & b_{2} & 0 & \cdots & 0 & 0 \\
0 & b_{2} & a_{3} & b_{3} & \cdots & 0 & 0 \\
\vdots & \vdots & \vdots & \vdots & \vdots & \vdots & \vdots \\
b_{N} & 0 & 0 & 0 & \cdots & b_{N-1} & a_{N}
\end{array}\right]
$$

where $a_{k}=-\left(b_{k}+b_{k-1}\right)$ for $k=1, \ldots, N$ (take $b_{0} \equiv b_{N}$ ), and the values of $b_{k}$ can be randomly sampled from uniform distribution $U[0,1]$. For each particular realization of $G$, calculate the largest eigenvalue $\lambda$ and the corresponding eigenvector $\left\{u_{j}\right\}_{j=1}^{N}$. The eigenvector has sharply localized amplitude peak at certain node and decay exponentially away.

In the simulation below, we will fix $N=100, \alpha=0.01 \mathrm{~s}^{-1}$. The initial synchronized state is obtained in the following way: $\left\{\hat{\theta}_{j}\right\}_{j=1}^{N}$ is randomly sampled from a uniform distribution, and given $k$ and $G$, the $P_{j}$ 's can be solved from the steady-state version of Eq. (1).

The disturbance is realized by imposing a perturbation $\delta$ on the phase. According to master stability analysis [9], as the coupling strength increases, the mode with the largest eigenvalue become unstable first and the oscillators in the region of unstable mode desynchronize. So the initial disturbance takes a localized form with amplitude $\Delta$.

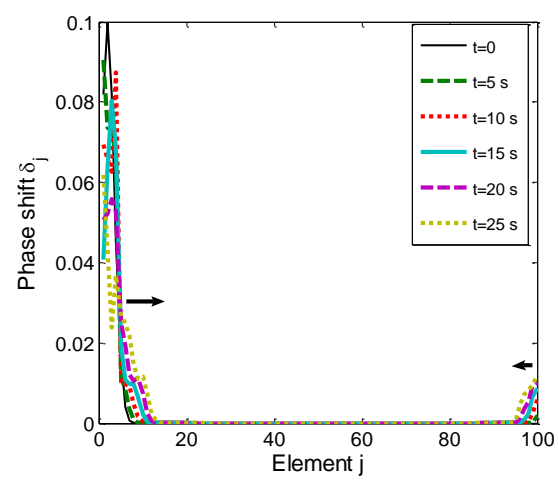

(a)

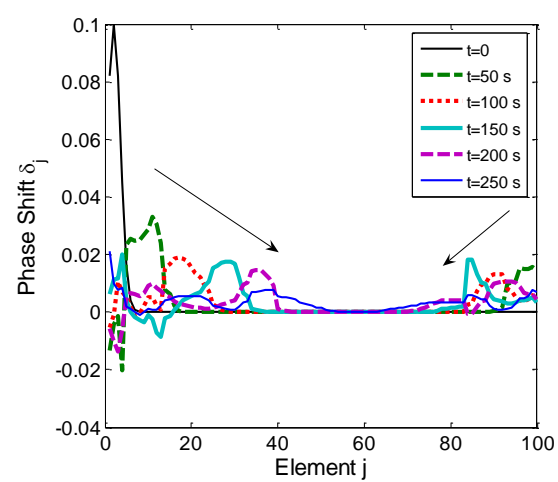

(b)

Fig. 2: Short term (a) and long term (b) dynamic distribution of the phase shifts of all oscillators for $k=0.1$ and $\Delta=0.1$. The arrows indicate the direction of the desynchronization wave propagation.

In Fig. 2, we show the snapshots of the distribution of the phase shifts of all oscillators at different time steps for $k=0.1$ (weak coupling) and $\Delta=0.1$. As seen from Fig. 2, the initial disturbance spreads to adjacent nodes as the amplitude decreases. In this case, the coupling strength between neighboring elements is 
weak, and essentially the dynamic behavior of Eq. (2) is damped oscillation. After sufficiently long time (> $500 \mathrm{~s}$ ), all elements will have essentially zero phase shift, i.e. back to the synchronized state.

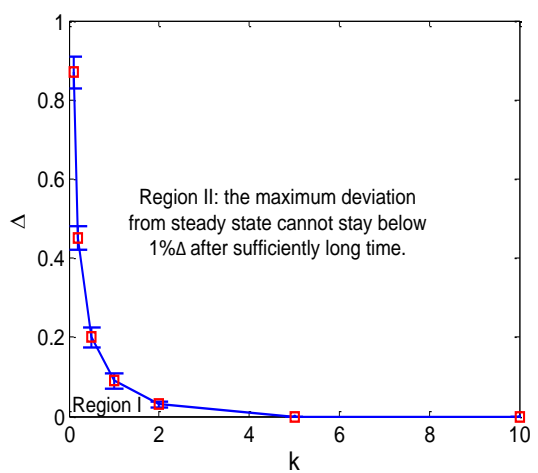

Fig. 3: Simulation results with various combinations of parameters $k$ and $\Delta$. The phase plane is divided into two regions: Region I (II) where the maximum deviation from steady state can (cannot) stay below $1 \%$ of the initial perturbation $\Delta$.

When the coupling strength exceeds some threshold, the desynchronized wave will quickly spread over the whole system. However, the long term behavior is distinct from that of weak coupling. There will be a persistent fluctuation in the distribution of phase shifts, which means that the system cannot return to synchronization anymore.

Fig. 3 presents the results of the simulations exploring the parameter plane of $(k, \Delta)$ and locating the boundary between the two above-mentioned regimes. As the coupling strength $k$ increases, smaller initial perturbation $\Delta$ is required to restore synchronization.

We also notice that, when the coupling strength is very high $(k \gtrsim 20)$, the system can be unstable and phase shift at some nodes may diverge. It remains unclear whether this is due to numerical error or dynamical instability.
Detailed theoretical analysis and numerical scheme will be presented in a future publication. The network topology in this section can be easily extended to more complex cases such as small world networks and Erdos-Renyi networks. However, our example is more mathematically tractable.

\section{Conclusions}

In summary, the Kuramoto-like oscillators can capture essential features of real systems, including wave-like propagation of desynchronization under disturbances. Based on a simplified model of power grid, we demonstrated the development of desynchronization and its relation with coupling strength. Although in previous works, strong coupling is required to realize a globally synchronized state, we find that when certain localized disturbance can spread over the whole network and will be persistent if the coupling is strong.

\section{References}

[1] M. Rohden, et al, Phys. Rev. Lett. 109, 064101 (2012).

[2] G. Filatrella, A. H. Nielsen, and N. F. Pederson, Eur. Phys. J. B 61, 485 (2008).

[3] F. Dorfler and F. Bullo, SIAM J. Control Optim. 50, 1616 (2012).

[4] J. A. Acebron, et al, Rev. Mod. Phys. 77, 137 (2005).

[5] A. Arenas, et al, Phys. Reports 469, 93 (2008).

[6] P. Kurdur, Power System Stability and Control (McGraw-Hill, New York, 1994).

[7] D. Butler, Nature 445, 586 (2007).

[8] E. Marris, Nature 454, 570 (2008).

[9] J. G. Restrepo, E. Ott, and B. R. Hunt, Phys. Rev. Lett. 93, 114101 (2004). 